\title{
A 6-bit 500-Ms/s Digital Self-Calibrated Pipelined Analog-to-Digital Converter
}

\author{
Yu-Hsun Chen and Tai-Cheng Lee \\ Department of Electrical Engineering \& Graduate Institute of Electronic Engineering \\ National Taiwan University, Taipei, Taiwan 10617, R.O.C.
}

\begin{abstract}
A 6-bit 500-Ms/s digital self-calibrated pipelined A/D converter is presented. Employing open-loop amplifiers in one bit conversion per stage architecture, the circuit operates in high speed and low power consumption. Using constant- $g_{m}$ biasing technique, the open loop amplifier is process-and-temperature insensitive. Comparator offset and full-scale error are removed by digital self-calibrated correction mechanism. Designed by a $0.18-\mu \mathrm{m}$ technology, the $\mathrm{A} / \mathrm{D}$ converter operates at $500-\mathrm{MHz}$ clock rate while dissipating $47 \mathrm{~mW}$. The FFT simulation result shows that the SNDR is $33.52 \mathrm{~dB}$ at $51-\mathrm{MHz}$ input frequency and 500-MSPS conversion rate.
\end{abstract}

Keywords: digital self-calibration, pipelined A/D converter

\section{INTRODUCTION}

High speed $A / D$ conversion plays an important role in many applications, such as automated test equipment, oscilloscopes and digital read channel systems [4, 5]. However, low power consumption and small area are more important than higher conversion rate and input bandwidth for these purposes [5]. The pipelined architecture offers power and/or speed advantages over flash or multistep approaches due to concurrent processing of analog signals [2]. Conventional pipelined ADCs mostly use electronic feedback to achieve highly linear and drift insensitive transfer characteristics in their conversion stages. Especially, the converter front-end amplifiers with large open-loop gain are needed to achieve the desired accuracy. Due to stringent design trade-offs between low noise and high bandwidth requirements, precision amplifiers dominate the power dissipation in most high speed designs [6]. A major limitation in all algorithmic converters is given by the inherent matching of the passive components, which are hard to control and have a significant effect on yield. Self-calibration and correction techniques have been developed to reduce the effects of component mismatches [2].

A 1-b-per-stage design is particularly amenable because each stage is very simple and fast [1]. In this paper, we employ open-loop amplifiers, operating in high speed and low power consumption to the $A / D$ converter in 1-bit conversion stage. Designed with constant $-G_{m}$ bias technique, the gain of the open-loop amplifier is determined by aspect ratio and is independent of process and temperature variation. The digital self-calibration architecture used here is a simple but effective mechanism, and tolerant of many nonidealities contributing to DNL and INL.

\section{ADC ARCHITECTURE}

Recently, the benefits of using open-loop structures in pipelined ADCs have been recognized and demonstrated. Using voltage-mode open-loop amplifier, the 12-bit ADC reported in [6] achieves $60 \%$ residue amplifier power savings over a conventional implementation. However, the amplification gain of open-loop differential pair depends significantly on process and temperature variation. In this work, we use an open-loop amplifier with a constant $-G_{m}$ bias topology. This bias circuit can track the $\mathrm{g}_{\mathrm{m}} \mathrm{R}$ constantly. Combining differential pair with source degeneration resistors, the voltage gain of the amplifier is linearized and can be expressed as

$$
\text { gain }=\frac{g_{m} R_{L O A D}}{1+g_{m} R_{S S}}
$$

While the $g_{m} R$ is hold constant, the gain of the amplifier can be determined by aspect ratio.

In pipelined $\mathrm{A} / \mathrm{D}$ converter design, power and speed optimization considerations favor the 1-bit conversion per stage approach [1]. In this case, the interstage gain is two and only one transition boundary occurs at the middle level of input in the transfer characteristic.

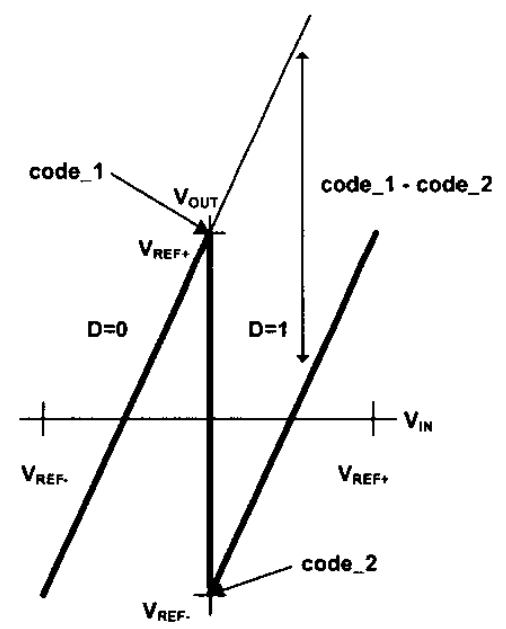

Fig. 1. Transfer characteristic for the 1-bit converter stage. 
Figure 1 shows that the transfer characteristic of 1-bit converter stage is suffered from process and circuit nonidealities. Including comparator offset, full-scale error, amplifier offset and component mismatches, they lead to linearity error in the ADC. The key feature of digital self-calibration is that the errors at the carry transitions are directly measured under the same condition as during the normal conversion. The missing decision levels cannot be removed alone but can be eliminated by using gain less than 2 and extra stages of pipeline which gives enough redundancy in the analog decision levels.

For each calibrated stage, the calibration is performed as follows: force an analog input value of $0 \mathrm{~V}$ (differential) and force the digital decision to the left and to the right of the transition. Then, the height of the analog transition in the transfer characteristic (Fig. 1.) is estimated in the digital domain with the rest of the pipeline, assumed calibrated. The calibration coefficient representing the height of that transition is stored with the corresponding raw data bit.

The architecture of the ADC is shown in the Fig. 2. The ADC consists of six stages with calibration mechanism and four extra stages for compensation.

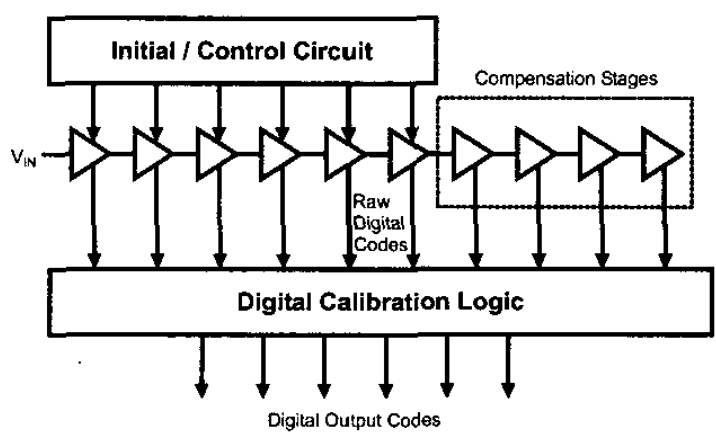

Fig. 2. The block diagram of ADC.

\section{BUILDING BLOCKS}

The architecture of a 1-bit conversion stage is shown in Fig. 3. With high input common voltage, only the pMOS pass gates with compensation dummy transistor is

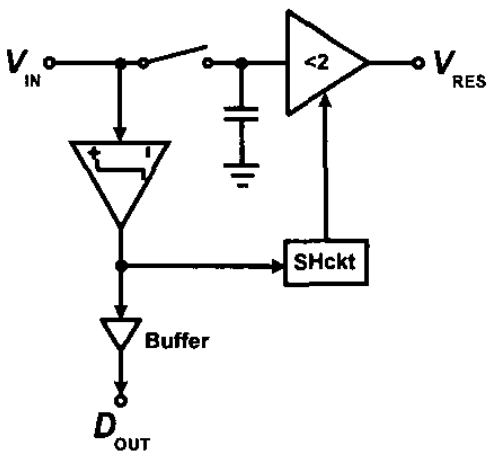

Fig. 3. 1-bit conversion stage architecture. inserted to operate as the $\mathrm{T} / \mathrm{H}$ circuit. The comparator senses the input polarity and generates a 1-bit digital code, driving the reference circuit to settle the reference voltage to be added or subtracted by the open-loop amplifier. Logic gates are built in both of the $\mathrm{T} / \mathrm{H}$ circuit and reference circuit to generate the valid control signal to calibrate the conversion stage. This design is compatibility with digital CMOS process since no MIM-caps are required.

\section{A. Constant- $G_{m}$ Bias Circuit}

The Fig. 4 shows the constant- $g_{m}$ bias topology. With . the identical dimensions of transistors $\mathrm{M}_{\mathrm{S}_{3}}$ and $\mathrm{M}_{\mathrm{S}_{4}}$, the PMOS devices require that current $\mathrm{I}_{\mathrm{OUT}}=\mathrm{I}_{\text {REF }}$. We can write $\mathrm{V}_{\mathrm{GS}, \mathrm{S} 1}=\mathrm{V}_{\mathrm{GS}, \mathrm{S} 2}+\mathrm{I}_{\mathrm{D}, \mathrm{S} 2} \mathrm{R}_{\mathrm{S}}$, or

$$
\sqrt{\frac{2 I_{\text {OUT }}}{\mu_{n} C_{O X}(W / L)_{N}}}+V_{T H, S 1}=\sqrt{\frac{2 I_{O U T}}{\mu_{n} C_{O X} K(W / L)_{N}}}+V_{T H, S 2}+I_{O U T} R_{S} .
$$

Because transistor $\mathrm{M}_{\mathrm{S2}}$ is a deep $\mathrm{N}$-well device, the body effect of transistor $M_{\mathrm{S} 2}$ can be neglected and hence

$$
I_{O U T}=\frac{2}{\mu_{n} C_{O X}(W / L)_{N} R_{S}^{2}}\left(1-\frac{1}{\sqrt{k}}\right)^{2}
$$

Thus, the transconductance of $\mathrm{M}_{\mathrm{S} 1}$ is equal to

$$
g_{m, S 1}=\frac{2}{R_{S}}\left(1-\frac{1}{\sqrt{K}}\right) \text {. }
$$

This equation is independent of temperature, process and supply voltage. The gain, $g_{m, s 1} R_{S}$, is equal to unity at $k=$ 4.

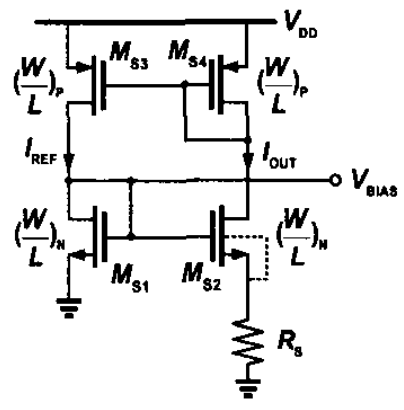

Fig. 4. Constant- $\mathrm{g}_{\mathrm{m}}$ bias topology.

\section{B. Proposed Open-Loop Circuit}

The proposed open-loop amplification circuit is shown in the Fig. 5. Two differential pairs with degeneration resistors constitute the open-loop amplifier. The nMOS transistors $\mathrm{M}_{3}, \mathrm{M}_{4}, \mathrm{M}_{7}$ and $\mathrm{M}_{8}$ in the deep $\mathrm{N}$-well are immune from the body effect. In the input differential pair, the bias transistors $\mathbf{M}_{1,2}$ and input transistors $\mathbf{M}_{3,4}$ are all chosen as $(\mathrm{W} / \mathrm{L})_{1,2,3,4}=2(\mathrm{~W} / \mathrm{L})_{\mathrm{S} 1}$, where $(\mathrm{W} / \mathrm{L})_{\mathrm{S} 1}$ 


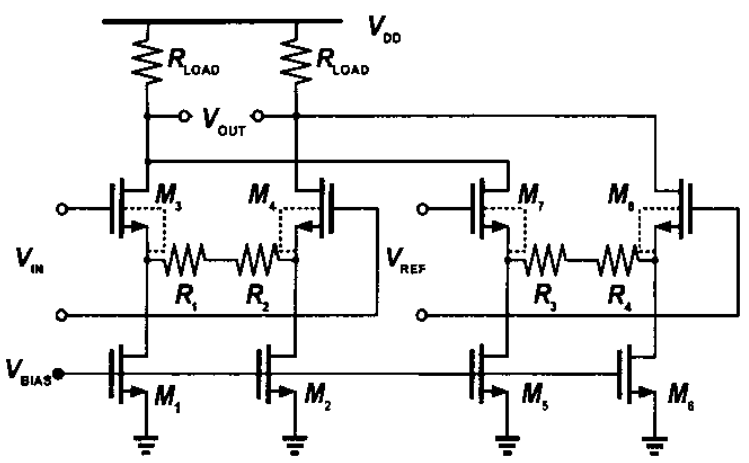

Fig. 5. Proposed open-loop amplifier.

is the size of the transistor $\mathrm{M}_{\mathrm{S} 1}$ of the bias circuit. Thus, the transconductance of the transistors $M_{3}$ and $M_{4}$ are two times of that of transistor $\mathrm{M}_{\mathrm{SI}}$. When the load resistors and the degeneration resistors are set to $R_{\mathrm{LOAD}}=$ $3 R_{S}$ and $R_{t, 2}=R_{S}$ in Fig. 3, the gain of output voltage versus input voltage can be expressed by the following equation:

$$
\frac{V_{\text {OUT }}}{V_{I N}}=\frac{g_{m 3,4} R_{L O A D}}{1+g_{m, 3.4} R_{\mathrm{i}, 2}}=\frac{2 g_{m, S 1} \cdot 3 R_{S}}{1+2 g_{m, S 1} R_{S}}=\frac{2 \times 3}{1+2 \times 1}=2
$$

In the reference differential pair, set the transconductance of $M_{7,8}$ to $g_{m 7,8}=g_{m, S 1}$ and the degeneration resistors $R_{3,4}$ to $R_{3,4}=2 R_{S}$. Then the gain of output voltage versus reference voltage satisfies the following equation.

$$
\frac{V_{O U T}}{V_{R E F}}=\frac{g_{m 7,8} R_{L O A D}}{1+g_{m 7,8} R_{1,2}}=\frac{g_{m, S 1} \cdot 3 R_{S}}{1+g_{m, S 1} \cdot 2 R_{S}}=\frac{1 \times 3}{1+1 \times 2}=1
$$

By summing equations (5) and (6), the output voltage can be expressed as

$$
V_{O U T}=2 V_{I N} \pm V_{R E F}
$$

As expected, the gain of the open-loop amplifier exhibits the same characteristic of $1-b$ conversion stage.

Inevitably, third-order distortion of differential pair compresses the nominal gain, causing it less than two. Nevertheless, the gain-compression characteristic here leads the conversion stage to tolerant nonidealities and the missing decision level can be removed by digital self-calibration mechanism. In order to keep the input transistors $\mathbf{M}_{3}, \mathbf{M}_{4}, \mathbf{M}_{7}$ and $\mathbf{M}_{8}$ operate in saturation region, the open- loop amplifiers run from a $2.5-\mathrm{V}$ power supply.

\section{Dynamic Comparator}

A fully differential dynamic comparator applied to this A/D converter is shown in the Fig. 6 . When the latch signal $\mathrm{V}_{\mathrm{LATCH}}$ is at $0 \mathrm{~V}$, the comparator turns off current source $M_{1}$ and operates in tracking mode. Only the outputs are reset to $V_{D D}$ by transistor $M_{8}$ and $M_{9}$. In latch mode, $V_{\text {LATCH }}$ going to $V_{D D}$, the outputs are disconnected from the positive supply and the current source $M_{1}$ begins to conduct to latch in the circuit composed with nMOS transistors $M_{4}$ and $M_{5}$ and pMOS transistor $M_{6}$ and $M_{7}$. The drain voltages of $M_{2}$ and $M_{3}$ are amplified to $V_{D D}$ or $V_{S S}$ and held as output signals $V_{\text {OUT }}$ and $V_{\text {OUT. }}$. Dynamic comparators are turned off when they are inactive so they can operate in ultra low power consumption. Furthermore, small area and high speed

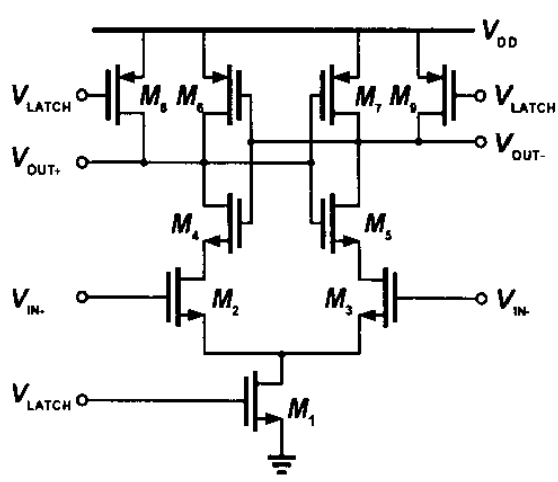

Fig. 6. Dynamic comparator circuit.

with low power consumption are realized using low $W / \mathrm{L}$ ratios for the transistors. This is because this dynamic circuit is free from the design constraint that a larger W/L ratio is needed for operation at the transistors in saturation region in the conventional current-biasing comparator circuit. Small sizes can be used for the transistors in the dynamic comparator circuit [4]. The comparator offset of the dynamic comparator is relative high and causes a shift of the major carry transition point. However, the carry transition is directly measured under the same condition with the digital self-calibration mechanism and the missing codes can be eliminated.

\section{SIMULATION RESULTS}

Figure 7 shows the static simulation of the INL and DNL with and without calibration. The peak INL is improved from 2.3 to $1.1 \mathrm{LSB}$ and the peak DNL is improved from 1.2 to $0.9 \mathrm{LSB}$. The missing decision levels are eliminated by the digital calibration.

Figure 8 shows the FFT spectrum of sine wave simulation with $51 \mathrm{MHz}$ input frequency and $500 \mathrm{MSPS}$ conversion rate. From Fig. 8 , the SFDR is $-40 \mathrm{~dB}$, the SNDR is $33.52 \mathrm{~dB}$ and the effective number of bit (ENOB) is 5.30 . The tone located on the $3^{\text {rd }}$ harmonica term comes from the nonlinearity of differential pairs [5]. At Nyquist rate input frequency, $244 \mathrm{MHz}$, the SNDR is reduced to $27.30 \mathrm{~dB}$.

The ADC is fabricated with $1 \mathrm{P} 6 \mathrm{M} 0.18-\mu \mathrm{m}$ CMOS process. The layout is shown in the Fig. 9 and the active area without digital correction circuit is $0.19 \mathrm{~mm}^{2}(0.68$ $\mathrm{mm} \times 0.28 \mathrm{~mm})$. The performance summary of the A/D converter is shown in the Table I. 

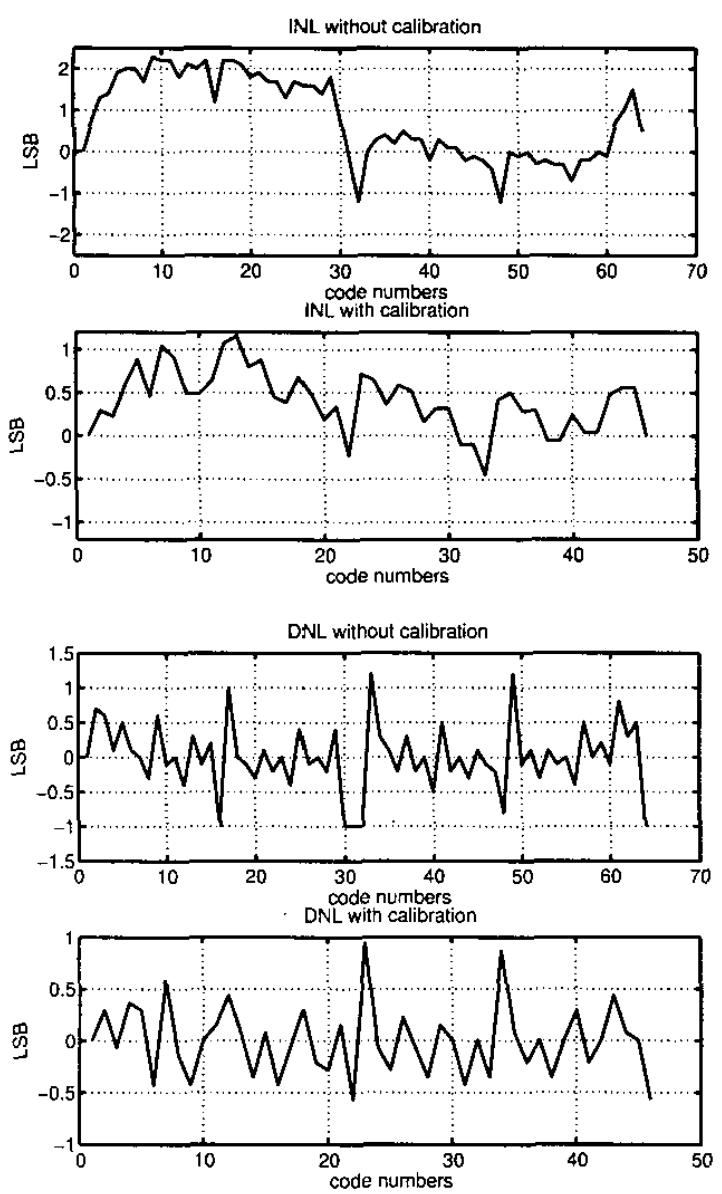

Fig. 7. The simulation result of the INL and DNL with and without calibration.

\section{ACKNOWLEDGEMENT}

The authors would like to thank National Chip Implementation Center (CIC) for supporting chip fabrication.

\section{REFERENCES}

[1] Andrew N. Karanicolas, Hae-Seung Lee and Kantilal L. Bacrania, "A 15-b 1-Msample/s Digitally Self-Calibrated Pipeline ADC," in IEEE J. Solid-State Circuits, Vol. 28, No. 12, December 1993.

[2] Ion E. Opris, Laurence D. Lewicki and Bill C. Wong, "A Single-Ended 12-bit $20 \mathrm{Msample/s}$ Self-Calibrating Pipeline A/D Converter," in IEEE J. Solid-State Circuits, vol. 33, No. 12, December 1998.

[3] Lauri Sumanen, Mikko Waltari and Kari Halonen, "A Mismatch Insensitive CMOS Dynamic Comparators for Pipeline $A / D$ Converters," in IEEE International Conference on Electronics, Circuits and Systems, Vol. 1, December 2000.

[4] Kouji Sushihara, Hiroshi Kimura, Yoichi Okamoto, Kazuko Nishimura and Akira Matsuzawa, "A 6b 800MSamples/s CMOS A/D Converter," in ISSCC Session 26, 2000.

[5] Koji Sushihara and Akira Matsuzawa, "A 7b 450MSample/s 50mW CMOS ADC in $0.3 \mathrm{~mm}^{2}$," in ISSCC, Session 10, 2002.

[6] Boris Murmann and Bernhard E. Boser, "A 12b 75MS/s Pipelined ADC using Open-Loop Residue Amplification," in ISSCC Session 18, 2003.

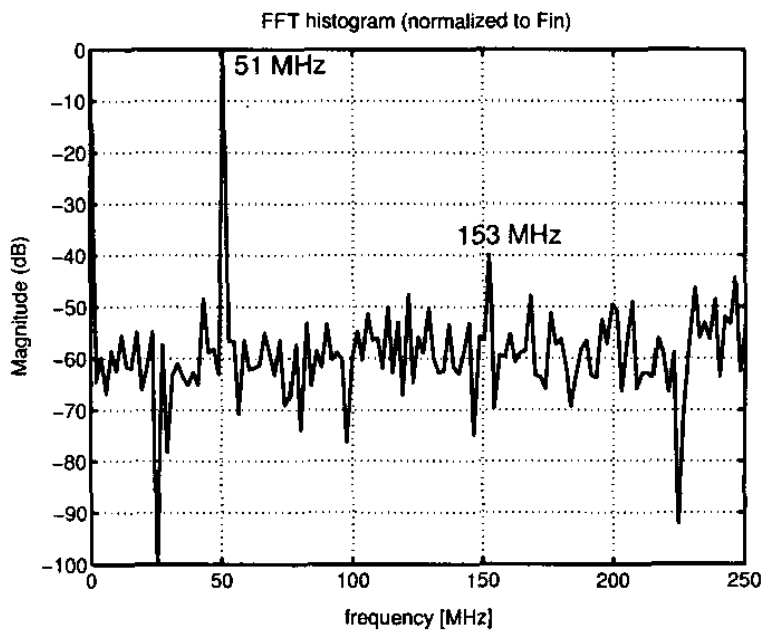

Fig. 8. The FFT spectrum with $51-\mathrm{MHz}$ input frequency and 500-MSPS.

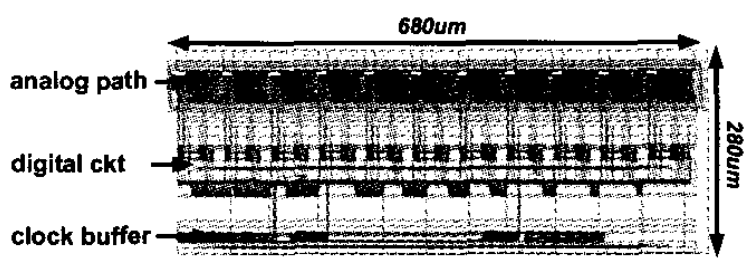

Fig. 9. The layout of the ADC.

Table I. The Summary of the ADC

\begin{tabular}{|c|c|}
\hline Technology & CMOS 0.18 1P6M \\
\hline Resolution & 6 bits \\
\hline Conversion Rate & $500 \mathrm{MSPS}$ \\
\hline Input Range & $800 \mathrm{mV}$ \\
\hline Supply Voltage & $1.8 \mathrm{~V} / 2.5 \mathrm{~V}$ \\
\hline $\begin{array}{c}\text { Power Consumption } \\
\text { w/o clock buffer }\end{array}$ & $34 \mathrm{~mW}$ \\
with clock buffer & $47 \mathrm{~mW}$ \\
\hline Chip Active Area & $0.19 \mathrm{~mm}^{2}$ \\
\hline DNL/INL & $<0.9 \mathrm{LSB} /<1.1 \mathrm{LSB}$ \\
\hline SNDR (500 MSPS) & \\
$51 \mathrm{MHz}$ & $33.52 \mathrm{~dB}$ \\
$244 \mathrm{MHz}$ & $27.30 \mathrm{~dB}$ \\
\hline
\end{tabular}

